\title{
Q Fever Osteomyelitis - Don’t Treat the Scan
}

\section{Thomas J Marrie*}

Department of Medicine, Dean Faculty of Medicine Dalhousie University, Canada

\section{Introduction}

$\mathrm{Q}$ fever is an infection caused by the micro-organism Coxiella burnetii [1]. It is a zoonosis with an extensive animal reservoir and it occurs in two forms acute and chronic [1]. The former consists of a variety of presentations including mainly self-limited febrile illness, pneumonia, hepatitis, and the latter endocarditis, endovascular infection and osteomyelitis [1-3]. There is very little clinical experience with Q fever osteomyelitis. This case report illustrates some of the difficulties encountered in managing such a patient.

\section{Case Report}

This 49 year old male school bus driver and hobby goat farmer became ill on March 4, 2004 with fever, headache, sore throat, cough, myalgias, anorexia and vomiting. Twelve days later he was admitted to his local hospital with a diagnosis of fever of unknown origin. Investigations here included negative blood cultures, elevated liver function tests and a normal chest radiograph. He remained febrile with daily temperatures of 38.5 to $39^{\circ} \mathrm{C}$. On the 10 hospital day, $22^{\text {nd }}$ day of his illness, he was transferred to a hospital in Edmonton. There a history of attendance at the delivery of pregnant goats in February was obtained and it was also noted that his daughter and wife had a febrile illness at about the same time as the onset of his illness. With this history antibody titres for C. burnetii infection were obtained and were consistent with recent infection with a phase II antibody titre of 1:2048 and a phase 1 titre of 1:512. Meantime he had been undergoing a workup for fever of unknown origin and computed tomographic scan of his abdomen showed splenomegaly with an infarct. A Gallium scan showed an area of focal uptake in the distal right tibia, although the radiographs of this area were normal (Figure 1). An MRI showed bone marrow edema consistent with early osteomyelitis (Figure 2). By now his fever had resolved and tjm saw him in consultation and recommended therapy for $\mathrm{Q}$ fever osteomyelitis. At this point he wanted to wait and see if his infection would resolve without therapy. He had increasing antibody titres on followup visits (Figure 3) and therapy with ciprofloxacin and rifampin was started with a subsequent decrease in antibody titres. He had severe symptoms of esophageal reflux trigged by these medications and he wished to stop therapy. Figure 3 shows the time course of his antibody titres and his subsequent treatment regime of doxycycline and hydroxychloroquine. These medications also resulted in a number of side effects and adherence was difficult and they were discontinued in March 2007 and then restarted December 2007 until November 2008. At that time his antibody titres were 1:128 and 1:512 for phase 1 and phase II respectively. As of March 2014 the antibody titres were 1:128 and 1:256 for phase 1 and II.

A follow up MRI of the distal tibia in December 2009 was essentially unchanged from previously and was interpreted as consistent with chronic osteomyelitis.

\section{Discussion}

Merhei et al. noted that up to 2012 only 20 cases of Q fever osteomyelitis had been reported [3]. Spondylodiscitis was present in 8 patients; long bones were involved in 6 , hip prosthesis in 2 , wrist in 2 and one each, talus and sacrum/ilieum. In some instances $Q$ fever

vertebral osteomyelitis is secondary to contiguous infection of an aortic graft [4]. The diagnosis is often delayed as exemplified by three pediatric cases reported by Noursi and colleagues [5]. The diagnosis is usually made serologically but in a few cases $C$. burnetii has been cultured from biopsy specimens of bone or from wound drainage. Amplification of C. burnetii DNA from specimens has been another diagnostic test [3].

Coxiella burnetii exists in two antigenic phases-phase I and phase II [6]. A variety of tests are available to detect antibodies to these antigens but the best one is the indirect immunofluorescence test [7]. In general in acute $\mathrm{Q}$ fever phase II antibodies are much higher than phase 1 antibodies while the reverse is true in chronic Q fever. Until recently most cases of chronic $Q$ fever meant endocarditis or vascular graft infection and a phase $1 \mathrm{IgG}$ antibody titer of 1:800 or greater by immunofluorescence was said to be diagnostic of $\mathrm{Q}$ fever endocarditis [8]. Our patient never had phased I titres higher than phase II but he did have a phase I titre of $>1: 800$.

Our patient differs from most of the cases in the literature in that he had osteomyelitis diagnosed during the acute phase of his illness. At that time he had bone marrow edema and if treatment had been started early there might have been resolution of the bone infection without going on to a more chronic phase with sequestrum formation. The treatment of choice for chronic $\mathrm{Q}$ fever is a combination of doxycycline and hydrochloroquine for 18 months for patients with native valve endocarditis [9]. We used the same strategy in treating our patient with osteomyelitis. The challenge then was when to stop therapy as

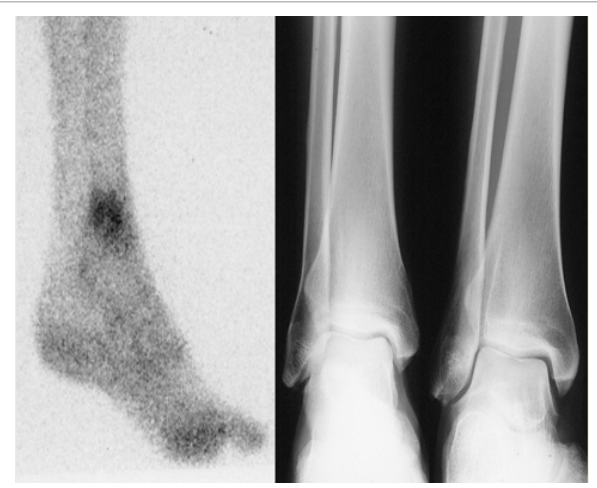

Figure 1: Radiographs of both distal tibia and fibulae (right) are normal and Gallium scan showing focal area of upatake (left) distal right tibia.

*Corresponding author: Thomas $\mathrm{J}$ Marrie, Department of Medicine Dalhousie University, 5849 University Avenue, Halifax, Nova Scotia, Canada, Tel: 902494 8099; Fax: 902494 7119, E-mail: t.marrie@dal.ca

Received December 23, 2014; Accepted February 22, 2015; Published February 24, 2015

Citation: Marrie TJ (2015) Q Fever Osteomyelitis - Don't Treat the Scan. J Med Microb Diagn 4: 178. doi:10.4172/21610703.1000178

Copyright: (c) 2015 Marrie TJ. This is an open-access article distributed under the terms of the Creative Commons Attribution License, which permits unrestricted use, distribution, and reproduction in any medium, provided the original author and source are credited. 


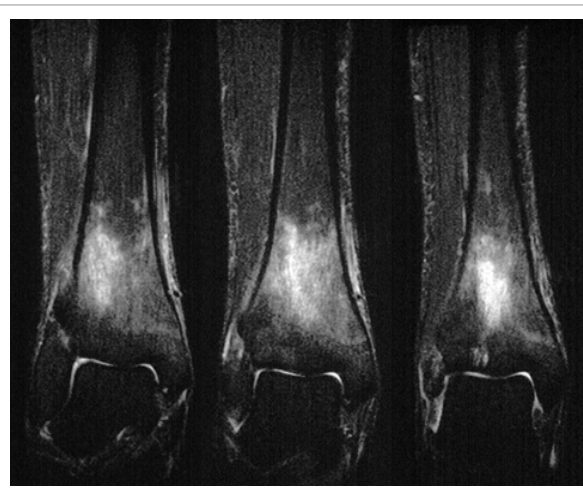

Figure 2: MRI distal right tibia showing marrow edema.

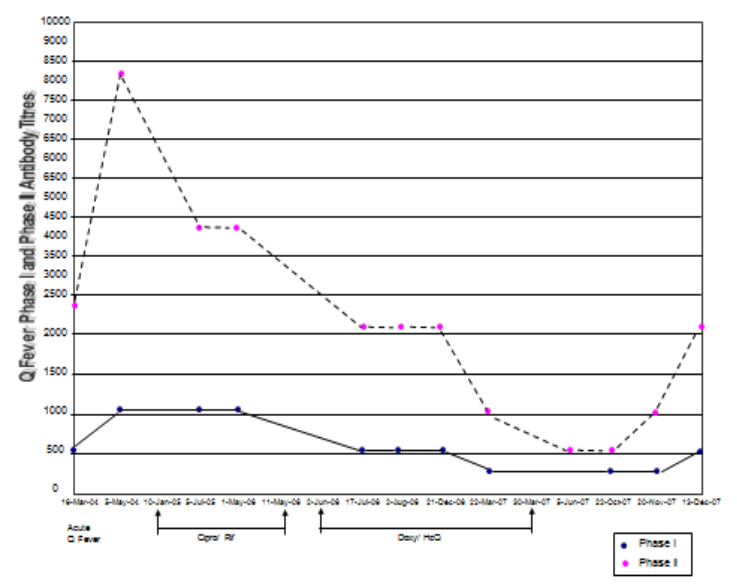

Figure 3: Serial antibody titres (reciprocal) phase 1 and phase II Coxiella burnetii.

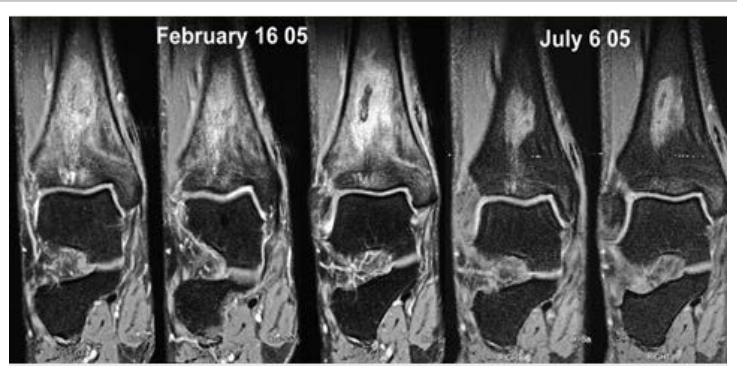

Figure 4: Follow-up MRI's of distal right tibia in February and July 2005 showing evolution to chronic change with sequestrum formation.

the MRI changes persisted. Clinically however he had no bone pain or tenderness and antibody levels were stable. Therapy was discontinued in March 2008 after almost four years of on again off again treatment. Six years later there has been no recurrence of his osteomyelitis.

Q fever osteomyelitis is an uncommon complication of a not uncommon infection in many countries. As more experience is gained with its management guidelines for treatment can be developed.

\section{References}

1. Sawyer LA, Fishbein DB, McDade JE (1987) Q fever: current concepts. Rev Infect Dis 9: 935-946.
2. Brouqui $P$, Dupont $H T$, Drancourt M, Berland $Y$, Etienne J, et al. (1993) Chronic $Q$ fever. Ninety-two cases from France, including 27 cases without endocarditis. Arch Intern Med 153: 642-648.

3. Merhej V, Tattevin P, Revest M, Le Touvet B, Raoult D, et al. (2012) Q fever osteomyelitis: A case report and literature review. Comp Immunol Microbiol Infect Dis 35: 169 -72.

4. Piquet $P$, Raoult D, Tranier P, Mercier C (1994) Coxiella burnetii infection of pseudoaneurysm of an aortic bypass graft with contiguous vertebral osteomyelitis. J Vasc Surg 19: 165-168.

5. Nourse C, Allworth A, Jones A, Horvath R, McCormack J, et al. (2004) Three cases of $Q$ fever osteomyelitis in children and a review of the literature. Clin Infect Dis 39: e61-66.

6. Q fever (1974) In: Christie AB (ed.) Infectious Diseases, Epidemiology and Clinical Practice. Churchill Livingstone, Edinburgh: 876-891.

7. Péter O, Dupuis G, Burgdorfer W, Peacock M (1985) Evaluation of the complement fixation and indirect immunofluorescence tests in the early diagnosis of primary Q fever. Eur J Clin Microbiol 4: 394-396.

8. Fournier PE, Casalta JP, Habib G, Messana T, Raoult D (1996) Modification of the diagnostic criteria proposed by the Duke Endocarditis Service to permit improved diagnosis of $Q$ fever endocarditis. Am J Med 100: 629-633.

9. Million M, Thuny F, Richet $H$, Raoult $D$ (2010) Long-term outcome of $Q$ fever endocarditis: a 26-year personal survey. Lancet Infect Dis 10: 527-535. 